\title{
Estudio de la actividad comparativa in vitro de telitromicina en patógenos respiratorios adquiridos en la comunidad en 13 centros clínicos chilenos
}

\author{
María T Ulloa F $F^{1 a}$, Rossanna Camponovo $C^{2}$, \\ Alejandra Fernández V3 ${ }^{3}$, Patricia García $C^{4}$, Valeria Prado J1, \\ Patricia González $A^{5}$, Pamela Rojas $S^{6}$, Jaime Inostroza $S^{7 b}$, \\ Mónica Lafourcade $R^{\mathbf{8}}$, Liliana Aguilera $A^{\mathbf{9 a}}$, Lorena Porte $\mathbf{T}^{10}$, \\ Carolina Cruz $\mathbf{P}^{11}$, Alejandro Joyas $\mathbf{M}^{12 a}$, Berta Alcaide $\mathbf{L}^{13 a}$, \\ María E Pinto $C^{1}$, María S Giglio $\mathbf{M}^{14 a}$.

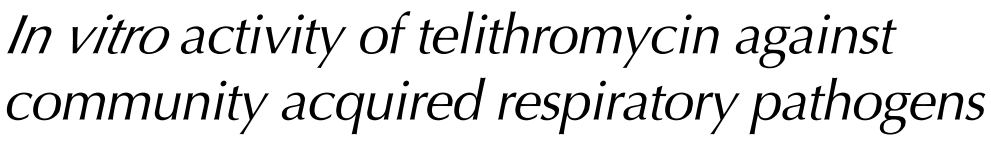

Background: Telithromycin is a new ketolide antimicrobial, that can be useful for the treatment of respiratory infections. Aim: To compare in vitro activity of telithromycin against respiratory pathogens, isolated in outpatient clinics. Material and methods: Two hundred eighty strains isolated from patients with respiratory infections, were studied. The strains studied were S pneumoniae, penicillin sensitive (SPNS:57); intermediate (SPNI:35), resistant (SPNR:25); S pyogenes (SP:57); H influenzae (HIN 51); M catarrhalis (MC:25) and S aureus meticillin sensitive (SAUS:30). Minimal inhibitory concentration (MC) by broth microdilution was studied for telitrhomycin and levofloxacin in all strains. Other antimicrobials studied, but not in all strains were erythromycin, clindamycin, trimetoprim sulphamethoxazole, oxacillin, amoxicillin-clavulanic acid and cefuroxime. Results: All strains were sensible to telithromycin at a concentration $\leq 4 \mu \mathrm{g} / \mathrm{ml}$. MC 90 and its range for SPNS was $0.03 \mu \mathrm{g} / \mathrm{ml}(\leq 0.004-0.12)$, for SPNI was $0.03 \mu \mathrm{g} / \mathrm{ml}(\leq 0.004-025)$, for SPNR was $0.06 \mu \mathrm{g} / \mathrm{ml}$ $(\leq 0.004-0.25)$, for HIN was $2 \mu \mathrm{g} / \mathrm{ml}(0.12-4)$, for SP was $0.5 \mu \mathrm{g} / \mathrm{ml}(\leq 0.004-2)$, for MC was 0.5 $\mu \mathrm{g} / \mathrm{ml}(0.06-2)$ and for SAU was $0.25 \mu \mathrm{g} / \mathrm{ml}(0.06-0.25)$. Conclusions: All studied pathogens were sensible to telithromycin in vitro. This antimicrobial is an alternative for the treatment of community acquired respiratory infections (Rev Méd Chile 2005; 133: 419-25).

(Key Words: Bacterial sensitivity tests; Ketolides; Respiratory tract infections; Telithromycin)

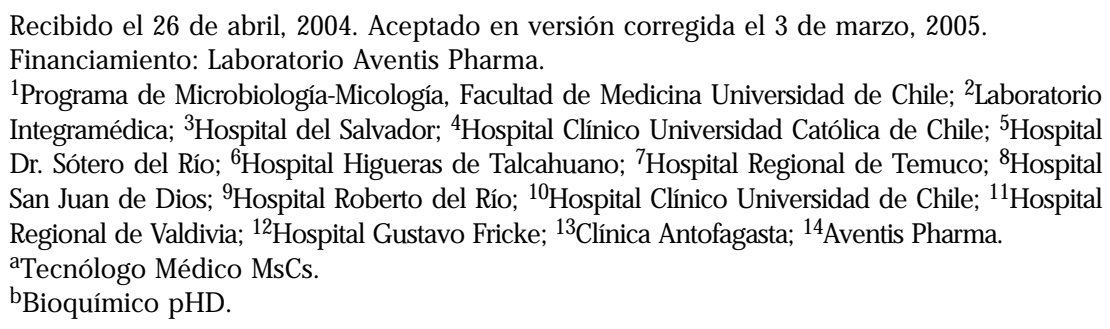

Correspondencia a: TM. María Teresa Ulloa F, MsCs. Programa de Microbiología y Micología, ICBM, Facultad de Medicina, Universidad de Chile. Independencia 1027, Clasificador 7, Independencia, Santiago-Chile. Fonos: 56-2-6786157-6786650. E mail: mtulloa@med.uchile.cl 
L as infecciones del tracto respiratorio son una _de las principales causas de mortalidad en el mundo; dentro de las causas bacterianas, los principales patógenos son: S pneumoniae, $\mathrm{H}$ influenzae, S aureus, M catarrhalis y S pyogenes. En menor proporción, patógenos atípicos como Mycoplasma pneumoniae, Chlamydiophila pneumoniae y Legionella pneumophila ${ }^{1}$.

Los patógenos más frecuentes del tracto respiratorio han adquirido resistencia a betalactámicos, macrólidos y lincosamidas, tanto a nivel nacional como mundial 2,3 , por ello, la industria farmacéutica ha desarrollado nuevas moléculas para enfrentar estos microorganismos, una de ellas es telitromicina. Pertenece a una nueva subclase de antimicrobianos de la familia de macrólidos-lincosamidas-estreptograminas, denominados ketólidos.

La principal característica química de la telitromicina es la ausencia de L-cladinosa en el anillo lactano, considerada durante largo tiempo esencial para la actividad antibacteriana, que fue removida y reemplazada por una función keto en la posición 3. Este grupo le confiere estabilidad en medio ácido, no induce resistencia $M L S_{\mathrm{B}}$ y es activo contra la mayoría de las cocáceas gram positivas que contiene el gen erm. Se adicionó, además, un grupo metoxi en posición 6, que junto con el grupo keto impide la ketalización interna que perjudica la absorción y ayuda a mantener la estabilidad del medio ácido $\mathrm{y}$, finalmente, se adicionó una cadena lateral aromática en C11-C12, lo que conduce a una óptima actividad antibacteriana y aumenta su fijación al ribosoma bacteriano, que es diez veces mayor que la eritromicina A en las cepas susceptibles a los macrólidos y 20 veces mayor cuando se combina con la función 3 keto del anillo lactano. La cadena lateral de arilaquil (grupo aromático unido a cadena de hidrocarburo), fijada en el residuo de C11-C12 carbamato, es responsable de características innovadoras in vitro, modo de acción, farmacocinética, farmacodinamia y buena entrada intracelular ${ }^{4-7}$.

En 1987 se describió por primera vez resistencia a penicilina en Spneumoniae en Chile, lo cual fue en $4,5 \%$ de las cepas estudiadas, no habiéndose detectado cepas resistentes a macrólidos ${ }^{8}$. Sin embargo, en 2000 se reportó $13,2 \%$ de resistencia a penicilina, $5,9 \%$ a cefotaxima y $15 \%$ a eritromicina particularmente entre las cepas con sensibilidad disminuida a penicilina ${ }^{9}$.
S pyogenes continúa siendo sensible a penicilina, pero su susceptibilidad a macrólidos ha sido variable en el tiempo con porcentajes que van de $0,5 \%$ en $1996^{10}$ hasta $7,2 \%$ en 199911,12 . Esta disminución en la sensibilidad se ha observado también en azálidos, tetraciclinas, clindamicina y trimetoprimsulfa.

En Estados Unidos de Norteamérica (USA) y en Europa se ha demostrado que telitromicina tiene una actividad sobre Haemophilus influenzae superior a la de macrólidos y es activa frente a Moraxella catarrhalis, Spneumoniae y S pyogenes así como en bacterias responsables de las neumonías atípicas (Legionella spp; Chlamydiophila pneumoniae; Chlamydia psittaci; Mycoplasma pneumoniae y Coxiella burnetti) ${ }^{4,13-18}$.

El objetivo de este estudio fue comparar la actividad in vitro de telitromicina con otros antimicrobianos en patógenos respiratorios, aislados de muestras clínicas, en diversos centros clínicos chilenos.

\section{MATERIAL Y MÉTODOS}

Se estudiaron 280 cepas (entre abril 2001 y septiembre 2002), de pacientes con infecciones respiratorias altas y bajas, incluyendo sólo una cepa por paciente. Las cepas fueron aisladas en los siguientes centros clínicos: Laboratorio Microbiología Integramédica (37 cepas), Hospital del Salvador (34 cepas), Hospital Clínico Universidad Católica de Chile (30 cepas), Facultad de Medicina, Universidad de Chile (30 cepas), Hospital Dr. Sótero del Río (27 cepas), Hospital Higueras de Talcahuano (27 cepas), Hospital Regional de Temuco (26 cepas), Hospital Roberto del Río (20 cepas), Hospital San Juan de Dios (18 cepas), Hospital Clínico Universidad de Chile (9 cepas), Hospital Regional de Valdivia (8 cepas), Hospital Gustavo Fricke (8 cepas) y Clínica Antofagasta (6 cepas). El tipo de muestras de las que fueron aisladas las cepas, se describe en la Tabla 1. Las especies, resistotipos y número de cepas analizados fueron: Spneumoniae penicilina sensible (SPNS:57); intermedio (SPNI:35) resistente (SPNR:25); S pyogenes (SP:57); H influenzae (HIN:51), M catarrhalis (MC:25) y S aureus meticilina sensible (SAUS:30). En cada centro se determinó la sensibilidad a telitromicina, además de los antimicrobianos de rutina por difusión en agar, según las normas del National Committee for 
Tabla 1. D istribución de 280 cepas aisladas de pacientes con infección respiratoria, según fuente de aislamiento

\begin{tabular}{|c|c|c|c|c|c|}
\hline Muestra & SPN & $\mathrm{SP}$ & SAU & HIN & MC \\
\hline Expectoración & 26 & & 10 & 10 & 13 \\
\hline Secreción faríngea & & 57 & & & \\
\hline Hemocultivo & 28 & & 1 & & \\
\hline Lavado broncoalveolar & 7 & & 1 & 6 & 1 \\
\hline Secreción ocular & 13 & & 3 & 10 & \\
\hline Secreción ótica por timpanocéntesis & 5 & & 4 & 7 & \\
\hline Líquido pleural & 2 & & 2 & & \\
\hline Secreción sinusal por punción & 13 & & 6 & 5 & 5 \\
\hline Secreción bronquial & 18 & & 3 & 10 & 5 \\
\hline Secreción traqueal & 5 & & & 3 & 1 \\
\hline Total & 117 & 57 & $\begin{array}{c}30 \\
280 \text { Cepas }\end{array}$ & 51 & 25 \\
\hline
\end{tabular}

Tabla 2. Actividad de telitromicina frente a diferentes microorganismos

\begin{tabular}{|llll|}
\hline Especie/resistotipo $^{*}$ & $(\mathrm{n})$ & CIM 90 & Rango $(\mu \mathrm{g} / \mathrm{ml})$ \\
\hline S pneumoniae/SPNS & $(57)$ & 0,03 & $(\leq 0,004-0,12)$ \\
S pneumoniae/SPNI & $(35)$ & 0,03 & $(\leq 0,004-0,25)$ \\
S pneumoniae/SPNR & $(25)$ & 0,06 & $(\leq 0,004-0,25)$ \\
S pyogenes & $(57)$ & 0,5 & $(\leq 0,004-2)$ \\
H influenzae & $(51)$ & 2 & $(0,12-4)$ \\
M catarrhalis & $(25)$ & 0,5 & $(0,06-2)$ \\
S aureus & $(30)$ & 0,25 & $(0,06-0,25)$ \\
\hline
\end{tabular}

*SPNS: S pneumoniae Penicilina Sensible, SPNI: S pneumoniae Penicilina Intermedia SPNR: S pneumoniae Penicilina Resistente.

Clinical Laboratory Standards (NCCLS) ${ }^{18}$. Las cepas fueron derivadas al Programa de MicrobiologíaMicología, de la Universidad de Chile, donde se determinó la concentración inhibitoria mínima (CIM) por microdilución en caldo (PML Microbiologicals ${ }^{\circledR}$ ) a telitromicina y levofloxacina a todas ellas; penicilina, eritromicina, clindamicina y trimetoprimsulfa a S pneumoniae (SPN) y SP; oxacilina, enitromicina, clindamicina y trimetoprimsulfa a SAU y ampicilina, amoxicilina/ácido clavulánico, trimetoprimsulfa y cefuroxima a HIN. Se determinó beta lactamasa por método de cefinasa a HIN y MC.

Se usaron controles de calidad de la American Type Culture Collection (ATCC) S aureus ATCC 29213, S aureus ATCC 25923, S pneumoniae ATCC 49619, H influenzae ATCC 49247. Los puntos de corte para los diferentes microorganismos fueron los aprobados por la NCCLS. Los valores utilizados para telitromicina fueron: S pneumoniae, Saureus y S pyogenes, sensible $\leq 1,0 \mu \mathrm{g} / \mathrm{ml}$, intermedia 2,0 $\mu \mathrm{g} / \mathrm{ml}$, resistente $\geq 4,0 \mu \mathrm{g} / \mathrm{m}, \mathrm{H}$ influenzae sensible $\leq 4,0 \mu \mathrm{g} / \mathrm{ml}$, intermedia $8,0 \mu \mathrm{g} / \mathrm{ml}$, resistente $\geq 16$ $\mu \mathrm{g} / \mathrm{ml}^{18-20}$. No existiendo punto de corte para $\mathrm{M}$ catharrhalis, se consideró sensible $\leq 2 \mu \mathrm{g} / \mathrm{ml}$, intermedia 4 y resistente $>8 \mu \mathrm{g} / \mathrm{ml}^{12}$.

\section{RESULTADOS}

Todas las cepas de $\mathrm{S}$ pneumoniae, $\mathrm{S}$ pyogenes, $\mathrm{H}$ influenzae, $\mathrm{M}$ catarrhalis y $\mathrm{S}$ aureus estudiadas fueron sensibles a telitromicina, registrándose los valores de CIM 90 y su rango respectivo $(\mu \mathrm{g} / \mathrm{ml})$ en la Tabla 2. En la Tabla 3a, se observa que de 
Tabla 3. Susceptibilidad de 117 cepas de Streptococcus pneumoniae aisladas del tracto respiratorio a diversos antimicrobianos

\begin{tabular}{|c|c|c|c|}
\hline Antimicrobiano & \% susceptibilidad & $\begin{array}{c}\text { CIM 50-CIM } 90 \\
\mu \mathrm{g} / \mathrm{ml}\end{array}$ & $\begin{array}{c}\text { Rango de } \\
\text { susceptibilidad } \\
\mu \mathrm{g} / \mathrm{ml}\end{array}$ \\
\hline Clindamicina & 98,3 & $0,03-0,12$ & $0,03-2$ \\
\hline Trimetoprimsulfa & 57,8 & $4-8$ & $0,12-8$ \\
\hline Levofloxacino & 100,0 & $0,5-1$ & $0,5-1$ \\
\hline Eritromicina & 89,5 & $0,03-0,06$ & $0,03-16$ \\
\hline Telitromicina & 100,0 & $0,008-0,03$ & $\leq 0,004-0,12$ \\
\hline \multicolumn{4}{|c|}{ Tabla 3b. Cepas de S pneumoniae con resistencia intermedia a penicilina $(n=35)$} \\
\hline Antimicrobiano & \% susceptibilidad & $\begin{array}{c}\text { CIM 50-CIM } 90 \\
\mu \mathrm{g} / \mathrm{ml}\end{array}$ & $\begin{array}{c}\text { Rango de } \\
\text { susceptibilidad } \\
\mu \mathrm{g} / \mathrm{ml}\end{array}$ \\
\hline Clindamicina & 94,2 & $0,03-0,06$ & 0,03-16 \\
\hline Trimetoprimsulfa & 48,6 & $0,5-4$ & $0,12-8$ \\
\hline Levofloxacino & 100,0 & $0,5-1$ & $0,5-1$ \\
\hline Eritromicina & 68,6 & $0,06-8$ & $0,03-64$ \\
\hline Telitromicina & 100,0 & $0,008-0,03$ & $\leq 0,004-0,25$ \\
\hline \multicolumn{4}{|c|}{ Tabla 3c. Cepas de S pneumoniae resistentes a penicilina $(n=25)$} \\
\hline Antimicrobiano & $\%$ susceptibilidad & $\begin{array}{c}\text { CIM 50-CIM } 90 \\
\mu \mathrm{g} / \mathrm{ml}\end{array}$ & $\begin{array}{c}\text { Rango de } \\
\text { susceptibilidad } \\
\mu \mathrm{g} / \mathrm{ml}\end{array}$ \\
\hline Clindamicina & 100 & $0,06-0,12$ & $0,03-0,12$ \\
\hline Trimetoprimsulfa & 56 & $0,25-8$ & $0,12-8$ \\
\hline Levofloxacino & 100 & $0,5-1$ & $0,5-2$ \\
\hline Eritromicina & 64 & $0,03-16$ & $0,03-64$ \\
\hline Telitromicina & 100 & $0,008-0,06$ & $\leq 0,004-0,25$ \\
\hline
\end{tabular}

las 57 cepas de $\mathrm{S}$ pneumoniae sensibles a penicilina, $89,5 \%$ presentaron sensibilidad a eritromicina, $57,8 \%$ a trimetoprimsulfa y $100 \%$ a levofloxacina y telitromicina. De las 35 cepas con sensibilidad intermedia a penicilina, se destaca que $68,6 \%$ fueron sensibles a eritromicina, $48,6 \%$ a trimetoprimsulfa y $100 \%$ a levofloxacino y telitromicina (Tabla 3b). De las 25 cepas resisten- tes a penicilina, $64 \%$ y $56 \%$ fueron sensibles a eritromicina y trimetoprimsulfa, respectivamente y todas fueron sensibles a levofloxacina y telitromicina (Tabla 3c).

$\mathrm{S}$ pyogenes fue $100 \%$ sensible a penicilina, levofloxacina y telitromicina; $93 \%$ y $89,5 \%$ a clindamicina y eritromicina, respectivamente (Tabla 4). H influenzae presentó una sensibilidad de 
80,4\% a ampicilina. Las 10 cepas resistentes a ampicilina fueron ß-lactamasa positiva y $100 \%$ de las cepas fueron sensibles a amoxicilina-ácido clavulánico y cefuroxima y telitromicina (Tabla 5). Noventa y cinco por ciento de las cepas de Moraxella catarrhalis fue resistente a ampicilina y betalactamasa positiva. Todas las cepas fueron sensibles a telitromicina y levofloxacina. Las cepas de $\mathrm{S}$ aureus meticilino sensible fueron $100 \%$ sensibles a telitromicina y levofloxacina, 98\% a trimetroprimsulfa y clindamicina y $60 \%$ sensible a eritromicina.

\section{DisCUSIÓN}

Las infecciones respiratorias bacterianas asociadas a patógenos adquiridos en la comunidad son un problema global, tanto por la morbilidad, como por la creciente resistencia antimicrobiana. Los patógenos más prevalentes en estas infecciones siguen siendo $\mathrm{S}$ pneumoniae, $\mathrm{H}$ influenzae, $\mathrm{S}$ pyogenes, $\mathrm{M}$ catarrhalis y $\mathrm{S}$ aureus.

Se han descrito, en el extranjero, numerosos mecanismos de resistencia antimicrobiana de estos patógenos. Así, el desarrollo de nuevos fármacos representa un desafío constante. En otro ámbito, en muchas ocasiones estos cuadros requieren un antimicrobiano de uso oral, especialmente en pacientes que son ambulatorios.

Estudios nacionales indican una situación similar a lo publicado en el extranjero, respecto de cifras crecientes de resistencia de estos patógenos a los antimicrobianos de uso habitual, especialmente S pneumoniae, que en los últimos años se destaca por su severidad e incidencia en nuestro medio ${ }^{8-11}$. Otro agente importante corresponde a $\mathrm{H}$ influenza, sin embargo, éste no ha sido tan estudiado desde el punto de vista de la resistencia.

Telitromicina ha mostrado tener buena actividad in vitro frente a estos patógenos. Las cepas incluidas en nuestro estudio representan centros relevantes de

Tabla 4. Susceptibilidad de 57 cepas de S pyogenes aislados de secreción faríngea a diversos antimicrobianos

\begin{tabular}{|lrcc|}
\hline Antimicrobiano & \% susceptibilidad & $\begin{array}{c}\text { CIM 50 -CIM } 90 \\
\mu \mathrm{g} / \mathrm{ml}\end{array}$ & $\begin{array}{c}\text { Rango de } \\
\text { susceptibilidad } \\
\mu \mathrm{g} / \mathrm{ml}\end{array}$ \\
\hline Penicilina & 100,0 & $0,06-006$ & $0,015-0,12$ \\
Clindamicina & 93,0 & $0,06-0,12$ & $0,03-32$ \\
Levofloxacino & 100,0 & $0,5-1,0$ & $0,12-2$ \\
Eritromicina & 89,5 & $0,03-0,06$ & $0,03-16$ \\
Telitromicina & 100,0 & $0,015-0,5$ & $\leq 0,004-2$ \\
\hline
\end{tabular}

Tabla 5. Susceptibilidad de 51 cepas de H influenzae aislados del tracto respiratorio a diversos antimicrobianos

\begin{tabular}{|lccc|}
\hline Antimicrobiano & $\begin{array}{c}\% \\
\text { susceptibilidad }\end{array}$ & $\begin{array}{c}\text { CIM 50-CIM } 90 \\
\mu \mathrm{g} / \mathrm{ml}\end{array}$ & $\begin{array}{c}\text { Rango de } \\
\text { susceptibilidad } \\
\mu \mathrm{g} / \mathrm{ml}\end{array}$ \\
\hline Ampicilina & 80,4 & 32 & $0,015-32$ \\
Amoxicilina/ácido clavulánico & 100,0 & $0,5-1$ & $0,006-4$ \\
Cefuroxima & 100,0 & $0,5-1$ & $0,015-1$ \\
Cloranfenicol & 96,1 & $0,5-1$ & $0,25-32$ \\
Trimetoprimsulfa & 65,0 & $0,25-8$ & $0,06-32$ \\
Levofloxacino & 100,0 & $0,015-0,12$ & $0,015-2$ \\
Telitromicina & 100,0 & $2-2$ & $0,12-4$ \\
\hline
\end{tabular}


nuestro país, aisladas de una amplia gama de manifestaciones clínicas del tracto respiratorio.

Las 280 cepas estudiadas resultaron ser sensibles a telitromicina con una CIM $\leq 4 \mu \mathrm{g} / \mathrm{ml}$, cifras que son similares a las anteriormente publicadas. Balfour et al, Bryskier y otros investigadores, han publicado que telitromicina es 2 a 5 veces más activa que claritromicina contra cocáceas gram positivas sensibles a eritromicina, tanto en Staphylococcus, $\mathrm{S}$ pneumoniae y $\mathrm{S}$ pyogenes ${ }^{4,6}$. Un hecho relevante es que telitromicina en Spneumoniae es activa independiente de su susceptibilidad a penicilina y eritromicina ${ }^{21-23}$. Nuestros resultados avalan esta observación, reflejada en una CIM 90 baja, independiente del resistotipo. La CIM 90 de las cepas de SPNS y SPNI fue de 0,03 $\mu \mathrm{g} / \mathrm{ml}$ y las cepas de SPNR presentaron una CIM 90 de $0,06 \mu \mathrm{g} / \mathrm{ml}$. Todas las cepas de $\mathrm{S}$ pyogenes siguen siendo sensibles a penicilina, no así a macrólidos, en que hay cepas resistentes que se han asociado con el consumo del antibiótico ${ }^{10-12,22,23}$. Las cepas de S pyogenes estudiadas en esta serie, 100\% fueron sensibles a telitromicina con CIM 50 de $0,015 \mu \mathrm{g} / \mathrm{ml}$ y CIM $90 \mathrm{de} 0,5 \mu \mathrm{g} / \mathrm{ml}$, a diferencia de la sensibilidad a eritromicina que fue de $89,5 \%$, con rangos de CIM 50 y CIM 90 de 0,03 y 0,06 $\mu \mathrm{g} /$ $\mathrm{ml}$, respectivamente (Tabla 3). Los mecanismos de resistencia para eritromicina en este tipo de cepas en nuestro país, han sido analizados por Palavecino y cols, no encontrándose diferencias con las cepas estudiadas en otros países ${ }^{11}$. La incorporación de la vacuna anti-Haemophilus influenzae tipo $b$, en nuestro país, en el esquema de vacunación nacional vigente, ha reducido drásticamente la presencia de este agente, sin embargo los HIN no capsulados siguen siendo aislados con gran frecuencia en las infecciones respirato-

\section{REFERENCIAS}

1. Baquero F, Barrett JF, Courvalin P, Morrisey I, Piddock L, Novick WJ. Epidemiology and mechanisms of resistance among respiratory tract pathogens. Clin Microbiol Infect 1998; 4(S2): 19-26.

2. Giglo M, Porte L, Herve B, Uloa MT. Susceptibilidad in vitro de patógenos respiratorios a levofloxacina y otros antimicrobianos. Rev Chil Infect 2000; 17: 18-24. rias $^{13,14}$. La actividad in vitro de telitromicina para Haemophilus influenzae ha sido probada en un gran número de cepas a nivel mundial. Los rangos de CIM 50 fluctúan desde 0,25 a $2 \mu \mathrm{g} / \mathrm{ml}$ y CIM 90 desde 0,5 a $4 \mu \mathrm{g} / \mathrm{ml}^{4,13,14}$. En nuestro estudio, HIN presentó una CIM $90 \mathrm{de} 2 \mu \mathrm{g} / \mathrm{ml}$, siendo todas las cepas sensibles. Con respecto a las cepas de HIN resistentes a ampicilina en todas ellas se detectó la presencia de $ß$ lactamasa.

Noventa por ciento de los aislamientos de Moraxella catharralis producen $ß$ lactamasa. La actividad in vitro de telitromicina tiene rangos de CIM 90 entre 0,03 y 0,5 $\mu \mathrm{g} / \mathrm{ml}$. Esto concuerda con los valores obtenidos en nuestro estudio, tanto en la producción de ß lactamasa 95\% como la CIM 90 a telitromicina de $0,5 \mu \mathrm{g} / \mathrm{ml}^{4,13,14}$.

Para Staphylococcus aureus con diferentes patrones de resistencia a meticilina y eritromicina, telitromicina ha demostrado una buena actividad in vitro con rangos de CIM 50 entre 0,06 y 0,12 $\mu \mathrm{g} / \mathrm{ml}$ y CIM 90 con rangos entre 0,12 y $0,25 \mu \mathrm{g} /$ $\mathrm{ml}^{4}$. En nuestro estudio, los valores obtenidos fueron entre 0,06 y $0,25 \mu \mathrm{g} / \mathrm{ml}$. La constante variación de la resistencia de los agentes etiológicos bacterianos a los antimicrobianos requiere información que puede servir como base para la aplicación de la terapia empírica a nivel local. Telitromicina aparece como una buena alternativa antimicrobiana en el manejo terapéutico de la infección respiratoria de la comunidad, especialmente $\mathrm{S}$ pneumoniae $24-26$.

Los datos obtenidos de este estudio adquieren relevancia, considerando que no siempre es posible contar con el diagnóstico etiológico de la IR, especialmente neumonía. Por lo tanto, es necesario contar con la información local al momento de la decisión terapéutica.

3. Nagal KN, Appelbaum P, Davies T, Keluy L, Hoeluman D, TAMBIC A ET AL. Susceptibilities to Telithromycin and Six other Agents and Prevalence of Macrolide Resistance due to L4 ribosomal Protein Mutation among 992 Pneumococci from 10 Central and Eastern European Countries. Antimicrob Agents Chemother 2002; 46: 371-7.

4. Balfour JA, FIgGitT DP. Telithromycin. Drugs 2001; 61: 815-29; discussion 830-1. 
5. Douthwaite S, Hansen L, Mauvais P. Macrolideketolide inhibition of MLS-resistant ribosomes is improved by alternative drug interaction with domain II of 23S rRNA. Mol Microbiol 2000; 36: 183-93.

6. BRYSKIER A. Ketolides-telithromycin, an example of a new class of antibacterial agents. Clin Microbiol Infect 2000; 6: 661-9.

7. Denis A, Agouridas C, Auger JM, Benedetti Y, Bonnefoy A, BRETIN F ET AL. Synthesis and antibacterial activity of HMR 3647. A new ketolide highly potent against erythromycin-resistant and susceptible pathogens. Bioorg Med Chem Lett 1999; 9: 3075-80.

8. Julet C, Giglo MS, Camponovo R. Sensibilidad de S pneumoniae y su importancia clínica. Rev Méd Chile 1987; 115: 852-7.

9. GonZÁlEZ P. Vigilancia de la resistencia a antimicrobianos. Rev Chil Infect 2002; 19 (S2): 135-9.

10. Giglo MS, Robles M, Rioseco ML, Vidal A, Segovia L. Streptococcus pyogenes: in vitro susceptibility to several antimicrobials two date periods. Rev Méd Chile 1996; 124: 715-9.

11. Palavecino EL, Riedel I, Berríos X, Bajaksouzian $S$, Jonson D, Kapalne et al. Prevalence and Mechanisms of macrolide resistance in Streptococcus pyogenes in Santiago, Chile. Antimicrob Agents Chemother 2001; 45: 339-41.

12. Vinagre C, Cifuentes M, Valdivieso F, Ojeda A, Prado V. Emergencia de resistencia a macrólidos en Streptococcus pyogenes. Rev Méd Chile 1999; 127: 1447-52.

13. Hoban D, Felmingham D. The PROTEKT surveillance study: antimicrobial susceptibility of Haemophilus influenzae and Moraxella catarrhalis form community-acquired respiratory tract infections. J Antimicrob Chemother 2002; 50: 49-59.

14. Wootton M, Bowker KE, JanowsKa A, Holt HA, Mac Gowan AP. In Vitro activity of HMR 3647 against Streptococcus pneumoniae, Haemophilus influenzae, Moraxella catarrhalis and B haemolytic streptococci. J Antimicrob Chemother 2002; 44: 445-53.

15. Biedenbach D, Barrett M, Jones RN. Comparative antimicrobial activity and kill curve investigations of novel ketolide antimicrobial agents (HMR 3004 and HMR 3647) tested against Haemophilus influenzae and Moraxella catarrhalis. Report USA 7967004/586. October 1997.

16. Caselias JM, Visser M, Mac Dougall N, Coco B, Tome G, Gliosca L, Grupo Colaborativo de Telutro-
MCINA Del Cono SuR. Multicenter study in southem South America of the in vitro activity of telithromycin in strains with defined resistance phenotypes isolated from community-acquired respiratory infections. Rev Esp Quimioter 2001; 14: 269-74.

17. LECLERQ R. Overcoming antimicrobial resistance: profile of a new ketolide antibacterial, telithromycin. J Antimicrob Chemother 2001; 48: 9-23.

18. Pankuch GA, Visam MA, Jacobs MR. Susceptibilities of penicillin and erythromycin-susceptible and resistant pneumococci to HMR 3647 (RU 66647), a new ketolide, compared with susceptibilities to 17 other agents. Antimicrob Agents Chemother 1998; 42: 624-30.

19. National Committee for Cunical Laboratory StanDARDS. Performance Standards for Antimicrobial Susceptibility testing: Fourteenth Informational Supplement. M100-S14, vol. 24 (1); 2004. NCCLS. Villanova, Pa. USA.

20. BARRY ALK, FuCHS PC, BROWN SD. Interpretative criteria and quality control parameters for telithromycin disc difusion susceptibility tests. J Antimicrob Chemother 2001; 48: 121-5.

21. Dabernat H, Seguy M, Delmas C. In vitro activity of telithromycin against Haemophilus influenzae. Pathol Biol 2002; 50: 58-62.

22. Farrell D, Morrissey I, BaKker S, Felmingham D. Molecular characterization of macrolide resistance mechanisms among Streptococcus pneumoniae and Streptococcus pyogenes isolated from the PROTEKT 1999-2000 study. J Antimicrob Chemother 2002; 50: 39-47.

23. Jalava J, Kataja J, Seppala H, Huovinem P. In vitro activities of the Novel Ketolide Telithromycin (HMR 3647) against Erythromycin-Resistant Streptococcus Species. Antimicrob Agents Chemother 2001; 45: 789-93.

24. Fuchs P, BaRRY A, Brown S. In vitro activity of telithromycin against Streptococcus pneumoniae resistant to other antibiotics, including cefotaxime. J Antimicrob Chemother 2002; 49: 399-401.

25. Felmingham D, Zhanel G, Hoban D. Activity of the Ketolide antibacterial telithromycin against typical community-acquired respiratory pathogens. J Antimicrob Chemother 2001; 48: 33-42.

26. Zhanel GG, Hoban D. Ketolides in the treatment of respiratory infections. Expert Opin Pharmacother 2002; 3: 277-97. 